\title{
Estimation of 2-D Direction of Arrival with an Extended Correlation Matrix
}

\author{
Ferid Harabi, Hatem Changuel, Ali Gharsallah \\ feridsamia@yahoo.fr, Changuelh@yahoo.fr, ali.gharsallah@,fstrnu.tn \\ groupe d'électronique, laboratoire de physique de la matiére molle, Faculty of sciences, Tunis,2092, Tunisia.
}

\begin{abstract}
This paper presents a new subspace-based 2-D direction of arrival (DOA) estimation algorithm for narrowband sources with high-resolution localization capabilities. DOA estimation is achieved by using the noise-subspace eigenvectors of a new extended correlation matrix (ECM). A 2-L shape antenna array is proposed. Unlike common planar and circular arrays, the novel antenna array with this special geometry requires no pair matching between the azimuth and elevation angle estimation also this key can remove the drawbacks of estimation-failure problems. The performance of the proposed approach is examined by a simulation study. The simulation results show a good estimate performance.
\end{abstract}

Index Terms - DOA estimation, extended correlation matrix, 2-L shape antenna array

\section{INTRODUCTION}

$\mathrm{D}$ irection of arrival (DOA) estimation from the outputs of an array of sensors is an important and well-studied problem with many applications in radar, sonar, and wireless communications. Recent applications include array processing for wireless mobile communications at the base station for increasing the capacity and quality of these systems $[1,4,5]$.

A large number of DOA estimation algorithms and analytical performance bounds have been developed. Beamformer method which scans the main beam of array antenna is the most fundamental technique. The other techniques based on eigenvalue decomposition of array input correlation matrix are the Min-Norm method, MUSIC (Multiple Signal Classification) [11], and ESPRIT (Estimation of Signal Parameters via Rotational Invariance Techniques) $[12,13]$ have are attracted attention in the mobile communication system. The maximum likelihood estimator [2] provides optimum parameter estimation. Its computational complexity is extremely demanding. However, MUSIC is most well-known for its superresolution capability and simplicity, in addition, it has less computational complexity than maximum likelihood methods.
The problem of estimating the two-dimensional directions of arrival, namely, the azimuth and elevation angles, of multiple sources was the topic of several researches $[8,10]$.

In this paper, we present a new subspace-based 2-D direction of arrival estimation method based on the proprieties of an extended covariance matrix developed by Horn and Johnson in [14].

The DOA estimation performance of an array strongly depends on the number and locations of the array elements. A number of researchers have considered the design of arrays to achieve or optimize desired performance goals. Much of the array design literature is devoted to linear arrays [3]-[6]. For planar arrays, performance comparisons of some common array geometries are given in [7]-[9].

The number and locations of the elements in an array strongly affect the DOA estimation performance of the array system. That is why, in this paper we consider a 2-L shape array geometry design for "good" DOA estimation performance.

Tayem and Kwon in [15] have demonstrated the reason why the novel antenna array with this special geometry requires no pair matching between the azimuth and elevation angle estimation. They have also explained that this key can remove the drawbacks of estimation-failure problems, unlike common planar and circular arrays.

The rest of the paper is organized as follows. The data model is presented in section II. The new correlation matrix is presented in section III. Section IV presents the 2-D direction of arrival estimation algorithm. Section V shows simulation results and section VI makes conclusions.

\section{DATA MODEL}

Consider the two-L-shape uniform linear array (ULAs) in the $x-z$ and the $y-z$ planes shown in Fig. 1 with interelement equals $d$, using three array elements placed on the $\mathrm{x}, \mathrm{y}$ and $\mathrm{z}$ axes. Each linear array consists of $N$ elements. The element placed at the origin is common for referencing purposes. 
Assume that $\mathrm{K}$ signals $s(\mathrm{t})$ from far-field uncorrelated sources impinge on the array with the same wavelength $\lambda$. Such that $\mathrm{k}^{\text {th }}$ source has an elevation angle $\theta_{k}$ and an azimuth angle $\phi_{k}, k=1, \ldots, K$.

We put the complex base-band representation of the signal received by the $n^{\text {th }}$ element of one subarray as $x_{n}(t)$ $(n=1,2 \ldots N)$, the signal sources are far apart from the subarray.

The subarray output vector at the snapshot $t$ is then given by:

$$
\begin{aligned}
& X(t)=\left(x_{1}(t), x_{2}(t), \ldots, \quad x_{N}(t)\right)^{T} \\
& =\sum_{k=1}^{K} a\left(\theta_{k}, \phi_{k}\right) s_{k}(t)+\eta(t), \mathrm{t}=1, \ldots, P
\end{aligned}
$$

" $T$ " is the transpose, $\eta(t)$ is an $N$-dimensional complex white noise vector with mean zero and covariance $\sigma^{2} I, I$ is the identity matrix of size $N$ and $\boldsymbol{a}\left(\theta_{k}, \phi_{k}\right)$ is the steering vector defined by :

$a\left(\theta_{k}, \phi_{k}\right)=\left[\left(1, e^{-j \varphi_{k, 2}}, \ldots, e^{-j \varphi_{k, N}}\right)\right]^{T}$

with $\varphi_{k, n}$ depends on the position and the geometry of the subarray.

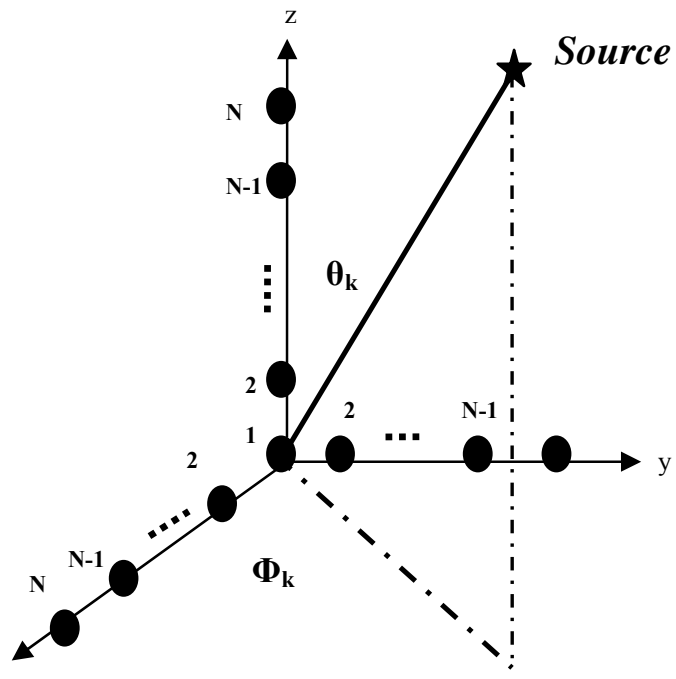

Fig.1: the 2-L-shape array configuration used for the joint azimuth and elevation $(\phi, \theta)$ DOA estimation.

\section{A- The steering vector of the $z$ axes subarray:}

Let $X_{z}(t)$ be the $N x I$ signal received at the linear subarray in the $\mathrm{z}$ axes at the snapshot $t$.

$X_{z}(t)=\left(x_{1 z}(t), x_{2 z}(t), \ldots, \quad x_{\mathrm{N} z}(t)\right)^{T}$
$=\sum_{k=1}^{K} a_{z}\left(\theta_{k}\right) s_{k}(t)+\eta_{k z}(t), t=1, \ldots \ldots, P$

where

$a_{z}\left(\theta_{k}\right)=\left[\left(1, e^{-j \varphi_{z k, 2}}, \ldots, e^{-j \varphi_{z k, N}}\right)\right]^{T}$

and $\quad \varphi_{z k, n}=\frac{2 \pi(n-1) d \cos \theta_{k}}{\lambda}$

$\theta_{k}$ is the elevation angle of the $\mathrm{k}^{\text {th }}$ source signal. $\eta_{k z}(t)$ is the additive White Gaussian noise of the $\mathrm{k}^{\text {th }}$ source signal in the $\mathrm{z}$ axes at the snapshot $t$.

\section{$B$ - The steering vector of the $x$ axes subarray:}

Let $X_{x}(t)$ be the $N x 1$ signal received at the linear subarray in the $\mathrm{x}$ axes at the snapshot $t$.

$$
\begin{aligned}
& X_{x}(t)=\left(x_{1 x}(t), x_{2 x}(t), \ldots x_{\mathrm{N} x}(t)\right)^{T} \\
& =\sum_{k=1}^{K} a_{x}\left(\theta_{k}, \phi_{k x}\right) s_{k}(t)+\eta_{k x}(t), t=1, \ldots \ldots, P
\end{aligned}
$$

where

$\left.a_{x}\left(\theta_{k}, \phi_{k x}\right)=\left[1, e^{-j \varphi_{x_{k}, 2}}, \ldots, e^{-j \varphi_{x_{k}, N}}\right)\right]^{T}$

and $\varphi_{x k, n}=\frac{2 \pi(n-1) d \sin \theta_{k} \cos \phi_{k, x}}{\lambda}$

\section{C- The steering vector of the $y$ axes subarray:}

Let $X_{y}(t)$ be the $N x 1$ signal received at the linear subarray in the y axes at the snapshot $t$.

$$
\begin{aligned}
& X_{y}(t)=\left(x_{1 y}(t), x_{2 y}(t), \ldots, \quad x_{\mathrm{N} y}(t)\right)^{T} \\
& =\sum_{k=1}^{K} a_{y}\left(\theta_{k}, \phi_{k y}\right) s_{k}(t)+\eta_{k y}(t), t=1, \ldots \ldots, P
\end{aligned}
$$

where 


$$
\begin{aligned}
& a_{y}\left(\theta_{k}, \phi_{k y}\right)=\left[1, e^{-j \varphi_{y_{k, 2}}}, \ldots, e^{-j \varphi_{y_{k, N}}}\right]^{T} \\
& \text { and } \varphi_{y_{k, n}}=\frac{2 \pi(n-1) d \sin \theta_{k} \sin \phi_{k, y}}{\lambda}
\end{aligned}
$$

\section{PREVIOUS WORK}

\section{III.1. The MUSIC algorithm:}

Let us review the procedure of the MUSIC algorithm [11]. The correlation matrix of sensor observations $X(t)$ is calculated:

$$
R=X(t) \cdot X(t)^{H}
$$

where $H$ represents a conjugate transpose. In practice, only a sample covariance matrix is available, i.e., an estimate of $R$ based on a finite number ( $P$ ) of data samples or snapshots

$$
\hat{R}=\frac{1}{P} \sum_{j=1}^{P} X\left(t_{j}\right) \cdot X\left(t_{j}\right)^{H}
$$

Then, obtain the eigenvalues decomposition $\hat{\mathrm{R}}=\mathrm{V} \Lambda \mathrm{V}^{\mathrm{H}}, \mathrm{V}=\left[\mathrm{v}_{1}, \ldots, \mathrm{v}_{\mathrm{N}}\right], \Lambda=\operatorname{diag}\left[y_{1}, \ldots, y_{N}\right]$, where $\mathbf{v}_{k}$ is an eigenvector ( $N$ - dimensional column vector) and $\mathrm{y}_{k}$ is the eigenvalue of $\mathbf{v}_{k}$ sorted as $y_{1} \geq \ldots \geq y_{K}$. The $K$ points where the function $U(\theta)=\sum_{k=K+1}^{N}\left|v_{k}{ }^{H} a(\theta)\right|^{2}$ approaches zero correspond to the directions $\theta_{1, \ldots,}, \theta_{K}$ of the source signals.

If the number $K$ of sources is smaller than the number $\mathrm{N}$ of sensors, all the signal components are represented in the signal subspace spanned by the first $K$ eigenvectors $\mathrm{v}_{1}, \ldots, \mathrm{v}_{\mathrm{N}}$, and the remaining $N-K$ eigenvectors $\mathrm{v}_{\mathrm{K}+1}, \ldots, \mathrm{v}_{\mathrm{N}}$ represents the noise subspace. The signal subspace and the noise subspace are orthogonal to each other since they are spanned by different eigenvectors.

The subspace spanned by the $\mathrm{K}$ steering vectors $\mathrm{a}\left(\theta_{1}\right), \ldots, \mathrm{a}\left(\theta_{K}\right)$ is also the signal subspace. When $\theta$ coincides with one of the source directions $\theta_{1}, \ldots, \theta_{K}$, the steering vector $\mathbf{a}(\theta)$ and the noise subspace $\mathbf{v}_{\mathrm{K}+1}, \ldots, \mathbf{v}_{\mathbf{N}}$ are orthogonal, and therefore $U(\theta)$ approaches zero. This is why source directions can be estimated using $U(\theta)$. For the noise subspace to exist, the number $N$ of sensors should be larger than the number $K$ of sources. Thus, the MUSIC algorithm is applicable for mixtures of up to $N-1$ signals.

III.2. The new extended correlation matrix:

In [14] a new correlation matrix is presented as:

$$
G^{\varphi}=\hat{R}+m a(\varphi) a(\varphi)^{H}
$$

where $\hat{R}$ is given in (13), $a(\varphi)$ is $1 \times \mathrm{N}$ array response vector for direction $\varphi$ given in (2) and $\mathrm{m}$ is a positive scalar. The matrix $G^{\varphi}$ is also positive definite and if $\mu_{k}$ and $e_{k}$ denote eigenvalues and eigenvectors of $G^{\varphi}$ respectively, $\mathrm{k}=1, \ldots, \mathrm{N}$,

Then it can be shown that while $m$ in (14) is a positive scalar we have [14]:

$$
\mu_{k} \geq y_{k} \quad \mathrm{k}=1, \ldots, \mathrm{N} \quad \forall \varphi
$$

Another important property of $G^{\varphi}$ is that when $\varphi$ in (14) is set to one of the source directions, i.e., $\varphi=\theta_{\mathrm{k}}$ for some $1 \leq k i \leq K$, then it can be shown that [14]

$$
\begin{aligned}
& \mu_{k} \geq y_{k} \quad k=1, \ldots, K \\
& \mu_{k}=y_{k}=\sigma_{n}^{2} \quad k=K+1, \ldots, N
\end{aligned}
$$

\section{THE NEW DIRECTION OF ARRIVAL ESTIMATION ALGORITHM}

We can note that except the actual source directions no other value of $\varphi$ have the properties showed in (15) and (16). The property stated in (16) does not depend on the value of scalar $m$ of (14) explicitly and will hold while $m$ is positive. Based on the above proprieties of the extended covariance matrix $G^{\varphi}$ and the 2-L shape arrays antenna an algorithm for estimating the direction of sources can be formed with the following steps:

A- Estimate of the elevation angle using the array in the $z$ axes:

1- From $P$ data samples compute $\hat{R}_{z}$ using the steering vector calculated in (4).

2- Compute $\hat{y}_{k z}, \mathrm{k}=1, \ldots, \mathrm{N}$, the eigenvalues of $\hat{R}_{z}$ that are in decreasing order.

3- Choose a positive $m$ and compute $G_{z}^{\varphi}$ for all possible values of $\varphi$.

4- For each value of $\varphi$, compute $\mu_{k z}^{\varphi}, \mathrm{k}=1, \ldots, \mathrm{N}$ the eigenvalues of $G_{z}^{\varphi}$ and calculate the function $D_{z}(\varphi)$ as: 


$$
D_{z}(\varphi)=\frac{1}{\sum_{k=K+1}^{N}\left(\mu_{k z}^{\varphi}-\hat{y}_{k z}\right)}
$$

5- Find all the values of $\varphi$ that maximize $D_{z}(\varphi)$. Elevation angles of sources $\theta_{1, \ldots,}, \theta_{K}$ are those values of $\varphi$ that correspond to $K$ largest maximums.

\section{$B$ - Estimate of the $x$ and $y$ axes array components of the} azimuth angle:

1- Using elevation angles of sources estimated previously and the steering vector of the signal received at the antenna subarray placed on the $\mathrm{x}$ axes calculated in (6), we compute $\hat{R}_{x}$ from $\mathrm{N}$ data samples.

2- Compute $\hat{y}_{k x}, \mathrm{k}=1, \ldots, \mathrm{N}$, the eigenvalues of $\hat{R}_{x}$ that are in decreasing order.

3- Choose a positive $m$ and compute $G_{x}^{\varphi}$ for all possible values of $\varphi$.

4- For each value of $\varphi$, compute $\mu_{k x}^{\varphi}, \mathrm{k}=1, \ldots, \mathrm{N}$, the eigenvalues of $G_{x}^{\varphi}$ and calculate the function $D_{x}(\varphi)$ in (18).

$$
D_{x}(\varphi)=\frac{1}{\sum_{k=K+1}^{N}\left(\mu_{k x}^{\varphi}-\hat{y}_{k x}\right)}
$$

5- Find all the values of $\varphi$ that maximize $D_{x}(\varphi)$. The $\mathrm{x}$ axes array component of the azimuth angles of sources $\hat{\phi}_{1 x}, \ldots, \hat{\phi}_{K x}$ are those values of $\varphi$ that correspond to $K$ largest maximums.

In the same way, we estimate the $\mathrm{y}$ axes array components of azimuth angles $\hat{\phi}_{1 y}, \ldots, \hat{\phi}_{K y}$ using the signal received at the antenna subarray placed on the y axes using the steering vector calculated in (10).

The azimuth angle estimation $\hat{\phi}_{k}$ can be written [15] as:

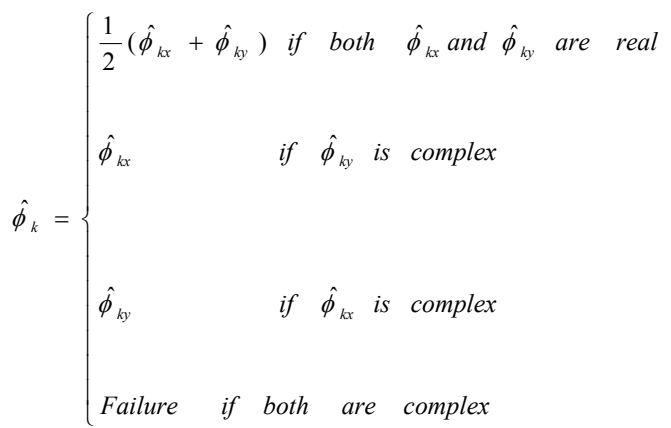

\section{Simulation results}

Computer simulations have been conducted to evaluate the 2-D DOA estimation performance of the proposed method. The parameters used in the simulation are as follows:

The sensors displacement $\mathrm{d}$ is taken to be half the wave length of the signal waves. We assume one single direct source $\mathrm{K}=1$, with direction of arrival DOA $(\theta, \phi)$. The additive noise is white Gaussian processes. The number of snapshots per trial is $\mathrm{P}=200$ and 1000 independent trials in total are used.

Figs. 2 and 3 show the histogram plots for the joint elevation and azimuth angles, respectively, for a single source with DOA located at $\left(70^{\circ}, 40^{\circ}\right)$ by using the proposed method of the 2-L shape array. We notice in each case that the method gives close elevation and azimuth DOA estimation and the clear peaks appear around $\left(70^{\circ}\right)$ and $\left(40^{\circ}\right)$, respectively.

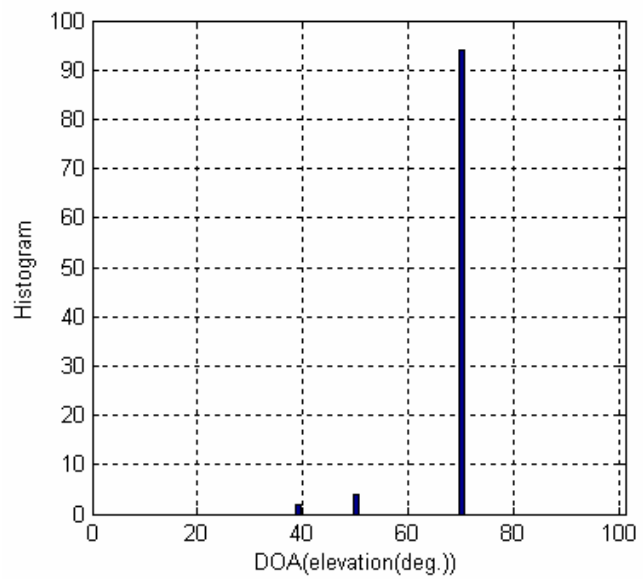

Fig.2: histogram of elevation angle for a single source located at $\left(70^{\circ}, 40^{\circ}\right), \mathrm{SNR}=10 \mathrm{~dB}$ by the proposed method with 5 elements on the $\mathrm{z}$ axes subarray

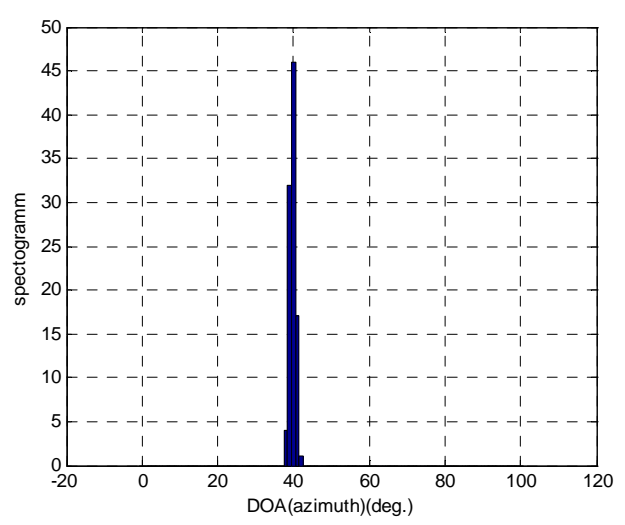

Fig.3: histogram of azimuth angle for a single source located at $\left(70^{\circ}, 40^{\circ}\right), \mathrm{SNR}=10 \mathrm{~dB}$ by the proposed method with 5 elements on the $\mathrm{z}$ axes subarray 
Figure 4 prove the performance of the proposed method and shows the spectrogram of the elevation and the azimuth angles for a single source located at $\left(90^{\circ}, 10^{\circ}\right)$ with $\mathrm{SNR}=10 \mathrm{~dB}$ and 5 elements on each subarray.

The 2-L shape configuration shows zero number of estimation failures for any pair of incident DOAs $(\theta, \phi)$ and all trials. The reasons why the proposed antenna array configuration shows no failures are discussed in [15].

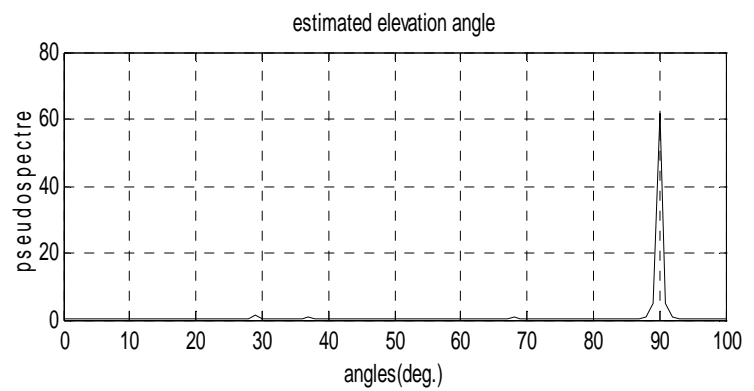

estimated azimuth angle

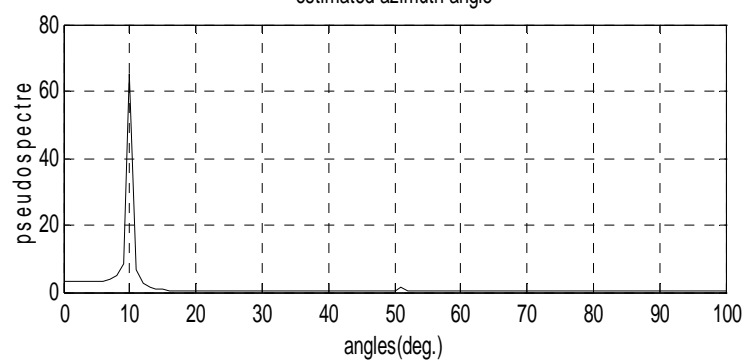

Fig..4. Pseudo spectre of elevation and azimuth angles for a single source located at $\left(90^{\circ}, 10^{\circ}\right), \mathrm{SNR}=10 \mathrm{~dB}$ by the proposed method with 5 elements on each subarray

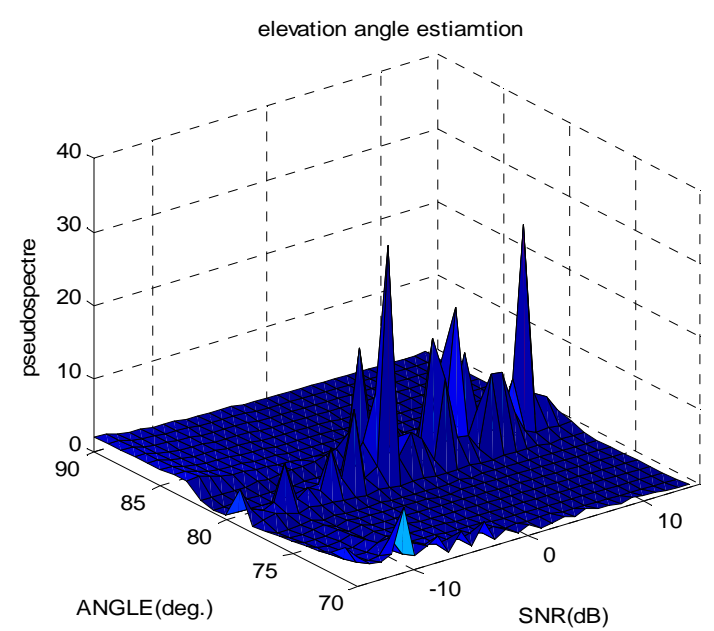

Fig.5. Elevation angle estimation with the proposed method of a single source located at $\left(80^{\circ}, 40^{\circ}\right)$ versus SNRs with 5 elements on the $\mathrm{z}$ axes subarray

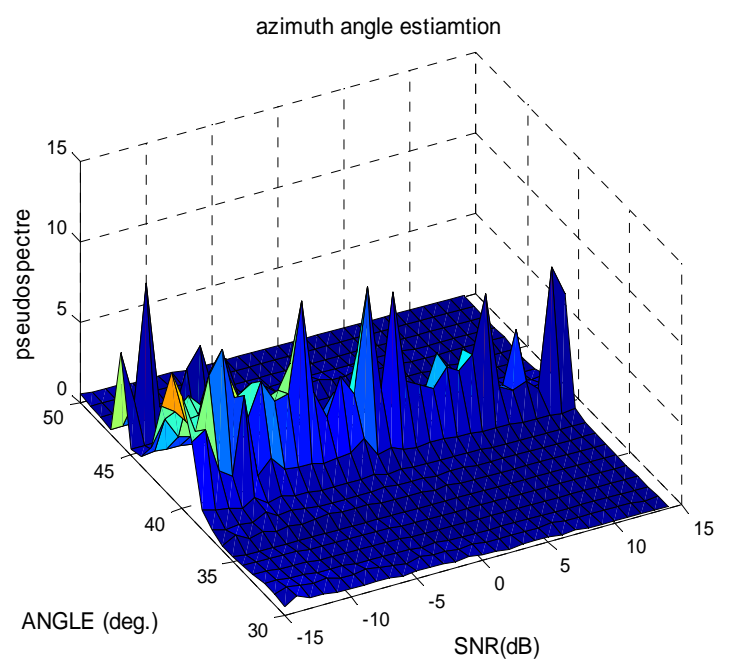

Fig.6. Azimuth angle estimation with the proposed method of a single source located at $\left(80^{\circ}, 40^{\circ}\right)$ versus SNRs with 5 elements on each subarray.

Figs. 5 and 6 show the spectrogram of elevation and azimuth angles, respectively versus SNRs in $\mathrm{dB}$ for a single source located at $\left(80,40^{\circ}\right)$. While comparing it to results of MUSIC method form figs 6 and 7 with the 2-L shape array, we observe that it gives good performances with low SNRs for the angle of elevation and we note a success of estimation in low SNRs for the azimuth angle better than MUSIC.

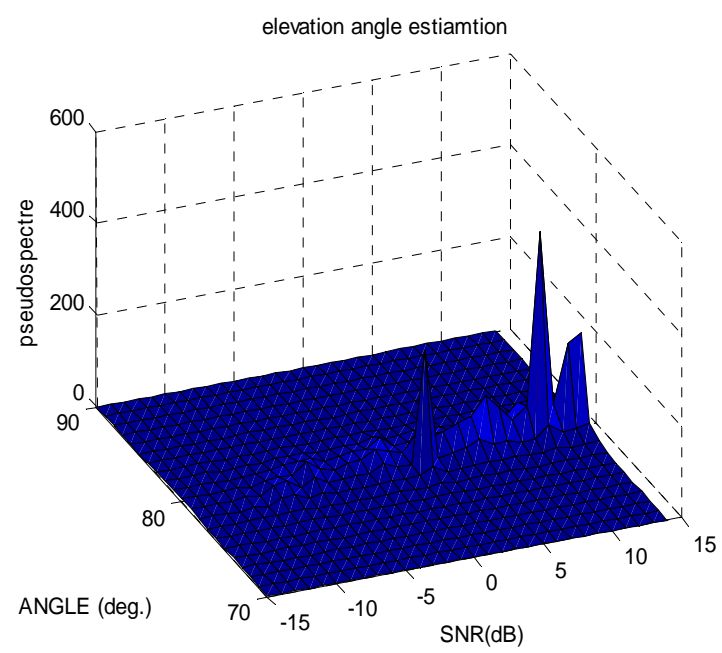

Fig .7. Elevation angle estimation with MUSIC method for a single source located at $\left(80^{\circ}, 40^{\circ}\right)$ versus SNRs with 5 elements on the $\mathrm{z}$ axes subarray. 


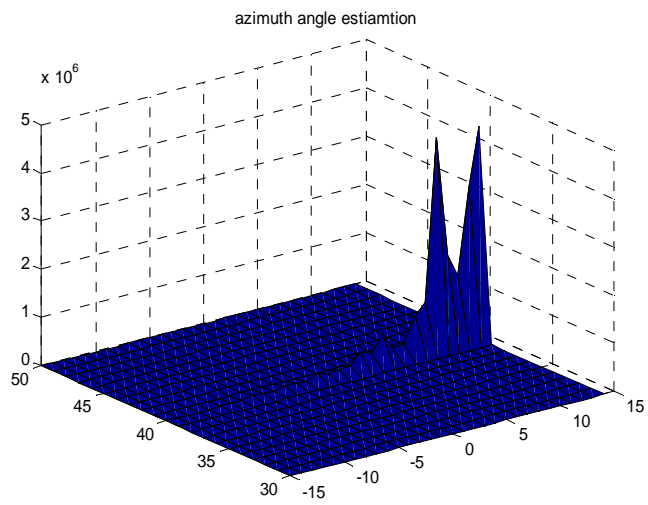

Fig .8. Azimuth angle estimation with MUSIC method for a single source located at $\left(80^{\circ}, 40^{\circ}\right)$ versus SNRs with 5 elements on each subarray.

The root mean square error (RMSE) of the proposed DOA estimation scheme was compared with that of the MUSIC and Propagator algorithms, using the 2-L shape array configuration in Fig.1. The RMSE for the joint DOA estimation is defined as:

$$
R M S E=\sqrt{E\left[\left(\hat{\theta}_{k}-\theta_{k}\right)+\left(\hat{\phi}_{k}-\phi_{k}\right)\right]}
$$

where $k$ represents the source index, $E[X]$ denotes the expectation of a random variable $X$, and $\left(\hat{\theta}_{k}, \hat{\phi}_{k}\right)$ are the pair of the elevation and azimuth angle estimates.

Fig. 9 shows the RMSE values of the joint elevation $\theta$ and azimuth $\phi$ DOA estimation versus the SNR in $\mathrm{dB}$ for the proposed method, MUSIC and Propagator method, respectively, when source signal arrive form direction located at $\left(90^{\circ}, 70^{\circ}\right)$. The total number of elements is 15 elements for the 2-L shape configuration for all methods. (five elements in each subarray).

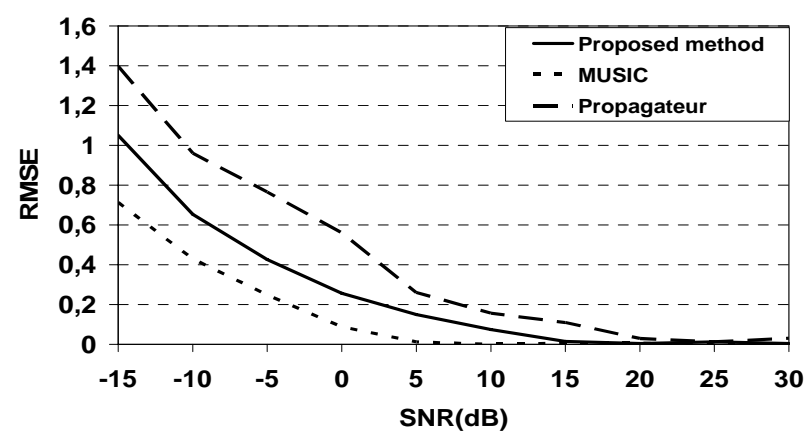

Fig.9. RMSE for a single source located at $\left(90^{\circ}, 70^{\circ}\right)$ with a 2-Lshape array antenna versus SNRs
We observe that the proposed method is $5.1 \mathrm{~dB}$ better than the Propagator method with the 2-L shape array and $\sim 3 \mathrm{~dB}$ less well than the MUSIC method with the same configuration.

\section{CONCLUSIONS}

A new method based on an extended correlation matrix with a 2-L shape antenna array configuration was proposed for the 2-D azimuth and elevation angle estimation problem. The proposed 2-D DOA estimation scheme shows a significant improvement. The proposed algorithm is quite general and can be applied to all array configurations. In other words, the proposed scheme does not require any pair matching for the 2-D DOA estimation problems, and the proposed algorithm shows no estimation failure for any pair of azimuth and elevation angles. Simulation results prove that the proposed scheme reduces the estimation error of both the azimuth and elevation angles and it proves a good performance at low SNRs.

\section{REFERENCES}

[1] J. Bakh Andersen, "Antenna arrays in mobile communications: gain, diversity, and channel capacity", IEEE Antennas Propag. Mag. 42 (2) 12-16, April 2001.

[2] Y. Hua, T. K. Sarkar, D. D. Weiner, "an L-shape array for estimating 2-D directions of wave arrival", IEEE Trans. Antennas Propagation 39(2) 143-146, 1991

[3] V. Murino, "Simulated annealing approach for the design of unequally spaced arrays", ICASSP International Conference on Acoustics, Speech, and Signal Processing, vol. 5, (Detroit, USA), pp. 627-3630, 1995.

[4] A. Kavak, "Adaptive antenna arrays for downlink capacity increase in third generation wireless CDMA", IEEE Radio and Wireless Conf., Boston, MA, pp. 77-80, August 2001.

[5] J. Razavilar, F. Rashid-Farrokhi, K. J. R. Liu, "TraMc improvements in wireless communication networks using antenna arrays", IEEE J. Selected Areas Commun. 18 (3) 458-471, March 2000.

[6] X. Huang, J. P. Reilly and M. Wong, "Optimal design of linear array of sensors", ICASSP International Conference on Acoustics, Speech and Signal Processing, vol. 2, (Toronto, Canada), pp. 1405-1408, 1991.

[7] J. W. Liang and A. J. Paulraj, "On optimizing base station antenna array topology for coverage extension in cellular radio networks", IEEE 45th Vehicular Technology Conference, vol. 2, (Stanford, CA), pp. 866-870, 1995.

[8] P. Krekel, E. Deprettre, "A two dimensional version of matrix pencil method to solve the DOA problem", European conference on circuit theory and design, pp.435-439, 1989.

[9] A. Manikas, A. Alexiou, and H. R. Karimi, "Comparison of the ultimate direction-finding capabilities of a number of planar array geometries", IEE Proceedings on Radar, Sonar and Navigation, pp. 321-329, 1997.

[10] Y. Wu, G. Liao, H. C. So "a fast algorithm for 2-D direction of arrival estimation", signal processing. 83 (8), p. 1827-1831, august 2003.

[11] R. O. Schmidt, "Multiple emitter location and signal parameter estimation", IEEE Trans. Antennas and Propagation AP-34 (3) 278-279, 1986.

[12] A. Paulraj, R. Roy and T. Kailath, “A subspace rotation approach to signal parameter estimation," Proc. IEEE, vol. 74, pp. 1044 1045, Jul. 1986.

[13] R. Roy and T. Kailath, "ESPRIT-estimation of signal parameters via rotational invariance techniques" IEEE Trans. Acoust., Speech, Signal Process., vol. 37, no. 7, pp. 984-995, Jul. 1989.

[14] R A. Horn and C. R. Johnson, "Topics in Matrix Analysis", Cambridge, U.K. : Cambridge Univ. Press, 1994. 
[15] N. Tayem, H. Kwon, "L-shape-2-D arrival angle estimation with propagator method”, IEEE Trans. vol.53.No5, May 2005. 